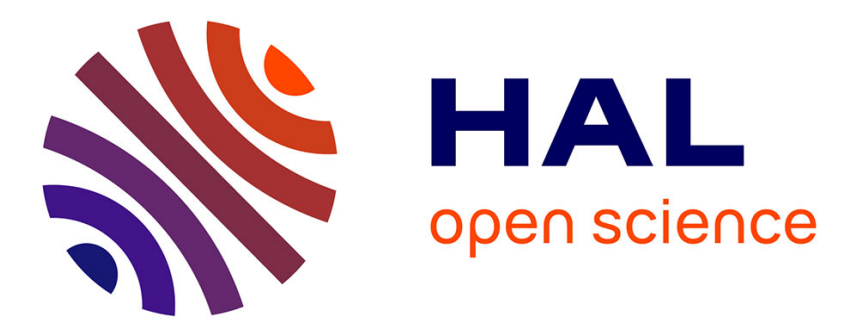

\title{
Heavy fragment emission in high energy reactions on heavy nuclei
}

\author{
G. Remy, J. Ralarosy, R. Stein, M. Debeauvais, J. Tripier
}

\section{To cite this version:}

G. Remy, J. Ralarosy, R. Stein, M. Debeauvais, J. Tripier. Heavy fragment emission in high energy reactions on heavy nuclei. Journal de Physique, 1970, 31 (1), pp.27-34. 10.1051/jphys:0197000310102700 . jpa-00206874

\section{HAL Id: jpa-00206874 https://hal.science/jpa-00206874}

Submitted on 1 Jan 1970

HAL is a multi-disciplinary open access archive for the deposit and dissemination of scientific research documents, whether they are published or not. The documents may come from teaching and research institutions in France or abroad, or from public or private research centers.
L'archive ouverte pluridisciplinaire HAL, est destinée au dépôt et à la diffusion de documents scientifiques de niveau recherche, publiés ou non, émanant des établissements d'enseignement et de recherche français ou étrangers, des laboratoires publics ou privés. 


\title{
HEAVY FRAGMENT EMISSION IN HIGH ENERGY REAGTIONS ON HEAVY NUGLEI
}

\author{
By G. REMY, J. RALAROSY, R. STEIN, M. DEBEAUVAIS et J. TRIPIER, \\ Département de Physique Corpusculaire, Centre de Recherches Nucléaires, Strasbourg.
}

(Reçu le 11 juillet 1969.)

\begin{abstract}
Résumé. - A l'aide de détecteurs visuels solides, nous avons étudié certaines réactions induites par des protons de haute énergie $(0,6 ; 3$; 18 ; et $23 \mathrm{GeV})$ dans des cibles d'uranium, de plomb et d'or.

Nous présentons des résultats concernant la fréquence relative de fission binaire et ternaire, la fréquence des événements ternaires coplanaires, les distributions angulaires et les distributions en masse et en impulsions concernant les fragments de fission ternaire.

Abstract. - Three prong events, registered in plastic visual detectors have been studied and calculated, and their cross-sections for various targets and various incoming energies have been compared to binary fission cross-sections.

Angular, energy and fragment mass distributions are also given.
\end{abstract}

Introduction. - During the last years, a process with nuclei desintegrating symmetrically into three fragments has been investigated by many authors $[1,2$, $6,8]$. This process can be induced by fast protons or heavy ions, the three emitted masses being in both cases of the same size. These ternary events have been called «Ternary fission ». This nuclear interaction must be distinguished from the low energy fission [3, 4, 5, 7] and from spontaneous fission of transuranium nuclei into two heavy fragments and a lighter one, for example lithium or helium.

Ternary fission cross-sections at high energies are between a few millibarn and some ten millibarns. We must note that the majority of these cross-sections have been obtained by solid state track detectors. Their use seems to be the most accurate technique to study ternary (and binary) fission at high energies. In this work, we emphasize ternary fission, but we shall also give some results concerning binary fission and fragmentation and point out the relative importance of these phenomena.

Gold, lead and uranium targets have been exposed to high energy protons of $0.6,3,18$ and $23 \mathrm{GeV}$ at C.E.R.N. and in Saclay. Our results concern :

1) Relative frequency of ternary to binary fission for various targets and for various energies.

2) The ratio of the number of coplanar ternary events to the total number of ternary events.

3) The angular distribution of two fragments for coplanar events.

4) Mass distribution for coplanar events. We have also calculated non coplanar events by means of some supplementary hypotheses.

5) Momentum distribution of the emitted fragments.

6) The distribution of total kinetic energy released.

Experimental. - Our experimental technique has already been published [1]. The detector used is a polycarbonate (makrofol). Its main property is a critical registration threshold of :

$$
\left(\frac{\mathrm{d} E}{\mathrm{~d} x}\right)_{\mathrm{c}}=8 \mathrm{MeV} / \mathrm{mg} / \mathrm{cm}^{2}
$$

If an ionizing particle has at the point of entrance into the detector an energy loss per unit path greater than this critical value, its track can be made visible by chemical treatment.

It can then be observed through an optical microscope. A standard development procedure is applied and one can detect in makrofol heavy particles with masses $M>16$.

The insensibility of this kind of detector to light particles allows the study of rare events involving heavy particles. The background which would be due to the much more frequent light particles, is not registered in plastics. In this way solid plastic detectors are ideal heavy particle detectors.

The sandwich technique used has a $4 \pi$ geometry. The target ( 30 to $50 \mu \mathrm{g} / \mathrm{cm}^{2}$ ) is evaporated on a $200 \mu \mathrm{m}$ plastic sheet $(30 \times 30 \mathrm{~mm})$. It is partially glued to another plastic foil and placed into a small polyethylene bag which is sealed under vacuum. This procedure insures good contact between detector and target. After irradiation, the target is dissolved and the tracks are developed with $5 \mathrm{~N} \mathrm{NaOH}$ at $60^{\circ} \mathrm{C}$ for $40 \mathrm{mn}$.

By observation with an optical microscope, we can distinguish 3 kinds of events.

A. Single tragks. - Their lenghts lie between 0 and approximatively $30 \mu \mathrm{m}$. They can be attributed to spallation or fragmentation reactions (we have taken in account only tracks which have projected horizontal lengths greater than $2.5 \mu \mathrm{m})$. The incoming protons enter the foil vertically. 
B. Two correlated tracks. - These tracks, generally due to two very heavy fragments, are correlated. They correspond approximately to one half of the mass of the target nucleus. This means that they originate from the same interaction. These events can be attributed either to binary fission or to double fragmentation. We can divide them into two very different classes :
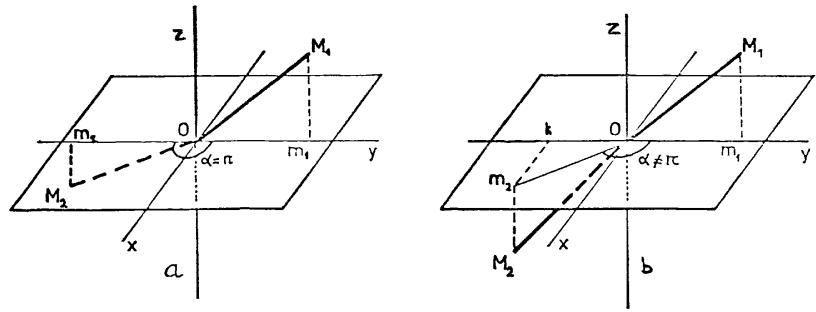

Scheme 1

a) If the trajectories of both heavy fragments and the incoming proton direction are coplanar, then the projections of $O M_{1}$ and $O M_{2}$ on a plane, perpendicular to the proton direction, are colinear. This implies that the momentum of the fissioning moving nucleus is in the proton direction.

b) If the projected angle of $O M_{1}$ and $O M_{2}$ is not $180^{\circ}$, then a transverse momentum component exists for the moving nucleus. This implies that lighter particles are produced. These particles are emitted during the cascade or evaporation step of the reaction and cannot be observed with the used detector.

G. Three correlated tracks (fig. 1). - Three tracks originate from the same target point. We have
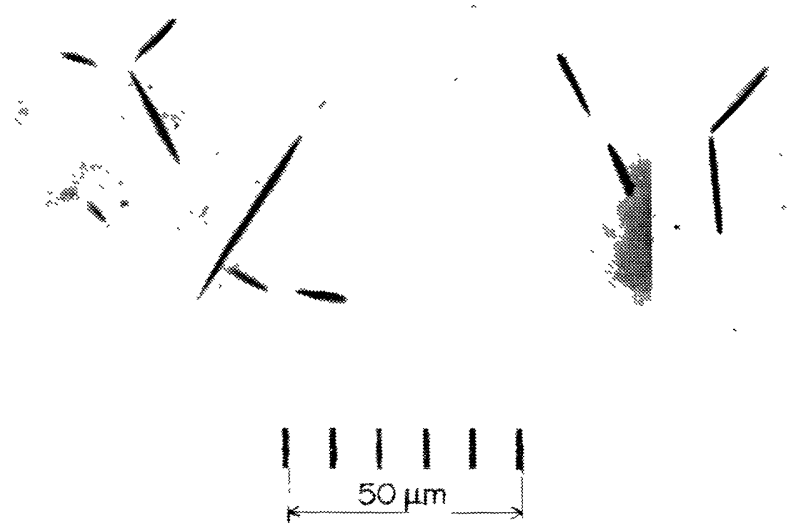

FIG. 1.

High energy proton interactions with heavy nuclei.

proved, that these events can only be genuine ternary events [1]. They are classified under the name "ternary fission", without implying a special mechanism of reaction (the dashed lines serves solely to recognize easily the corresponding points).

Results and discussions. - 1. Fragmentation. Figure 2 shows the $S / B$ ratio of single to binary tracks

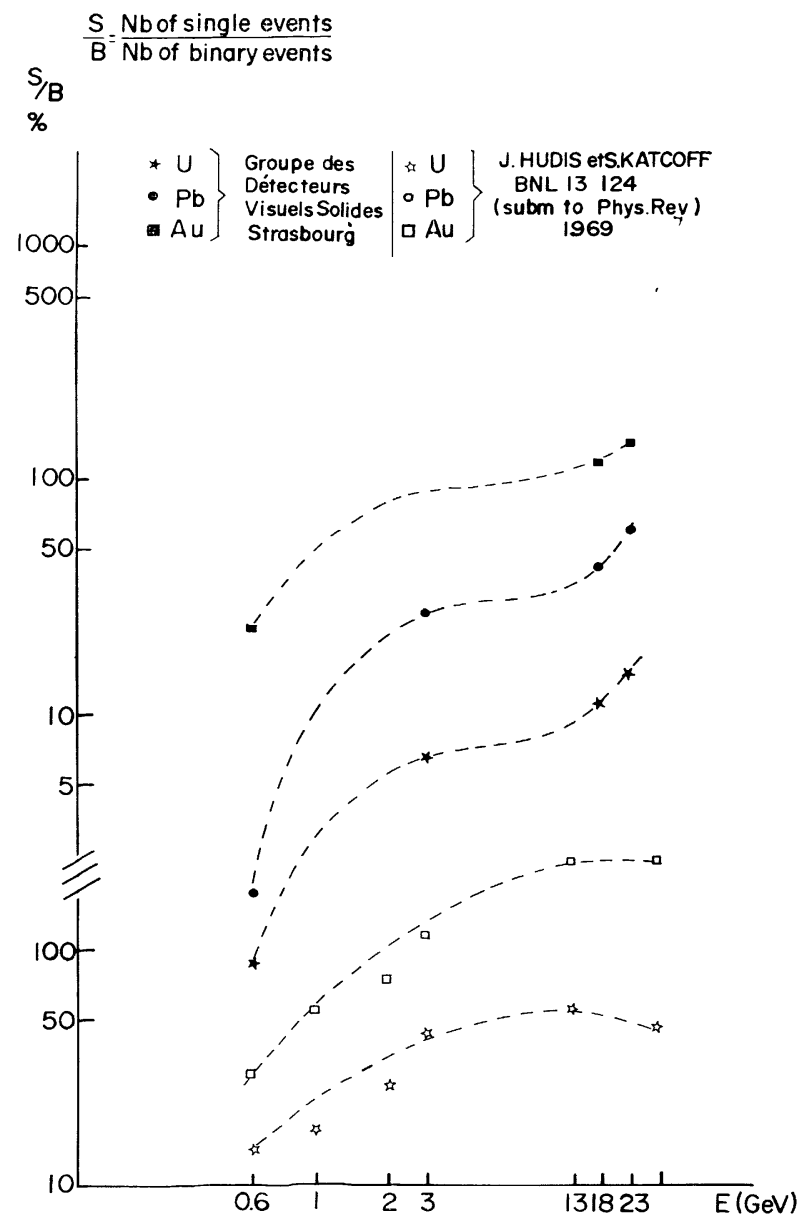

FIG. 2. - High energy proton interactions with uranium, lead and gold targets, ratio of single events to binary events.

as a function of target nucleus and incoming proton energy. It can be seen that :

a) For a given target nucleus, $S / B$ increases by a factor of 10 when the incoming proton energy rises from $0.6 \mathrm{GeV}$ to $25 \mathrm{GeV}$.

b) For a given incoming energy $S / B$ decreases when the target mass increases (the dashed lines serves solely to recognize easily the corresponding points).

We can compare our results to those obtained by Hudis and Katcoff [8]. They employed mica detectors and found a similar dependence for $S / B$. Our results are of the same order as theirs.

2. Ternary fission (fig. 1). - The ratio $T / B$ of ternary fission relative to binary fission is indicated in table I for every target nucleus and proton energy.

Figure 3 a shows $T / B$ versus incoming energy for lead, gold and uranium. It can be seen that :

a) For a given target nucleus, $T / B$ seems to have a maximum between 3 and $18 \mathrm{GeV}$ :

- that the value at $600 \mathrm{MeV}$ is very small and thence the uncertainty is large.

b) For a given energy the relative frequency of ternary events is a decreasing function of target mass. 


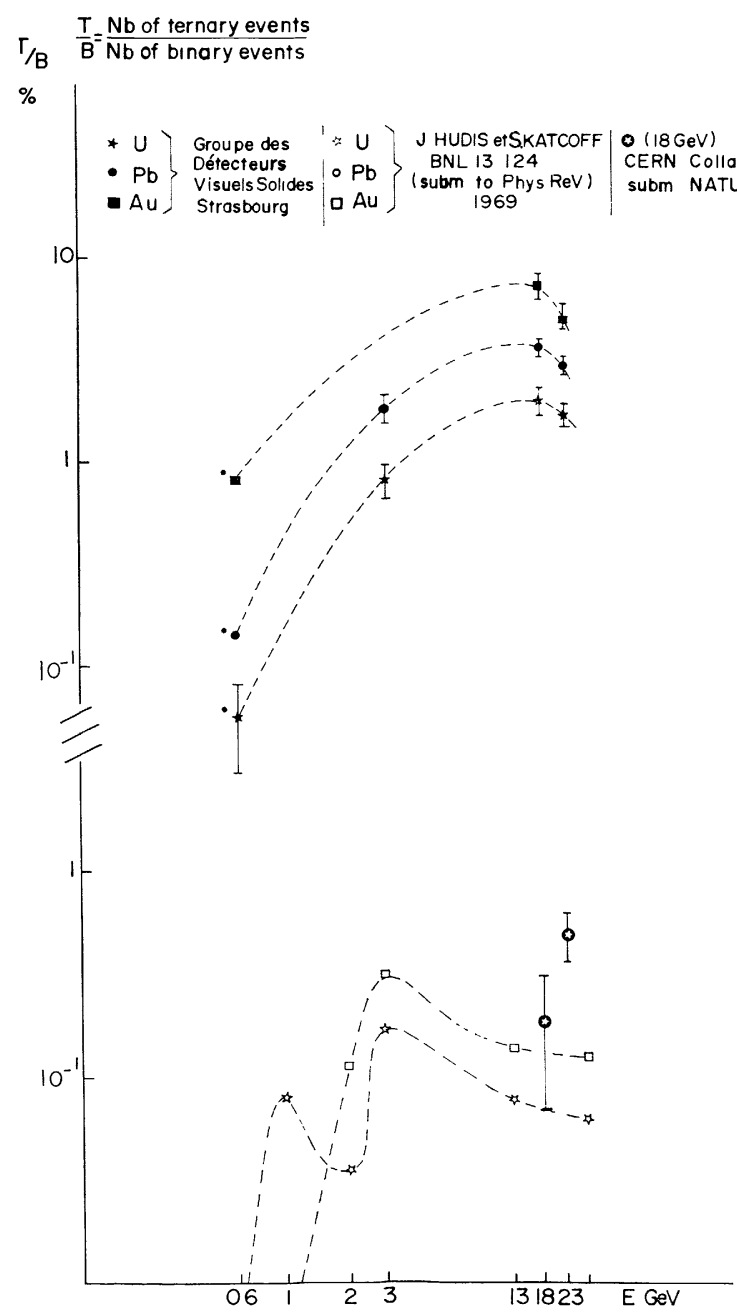

a
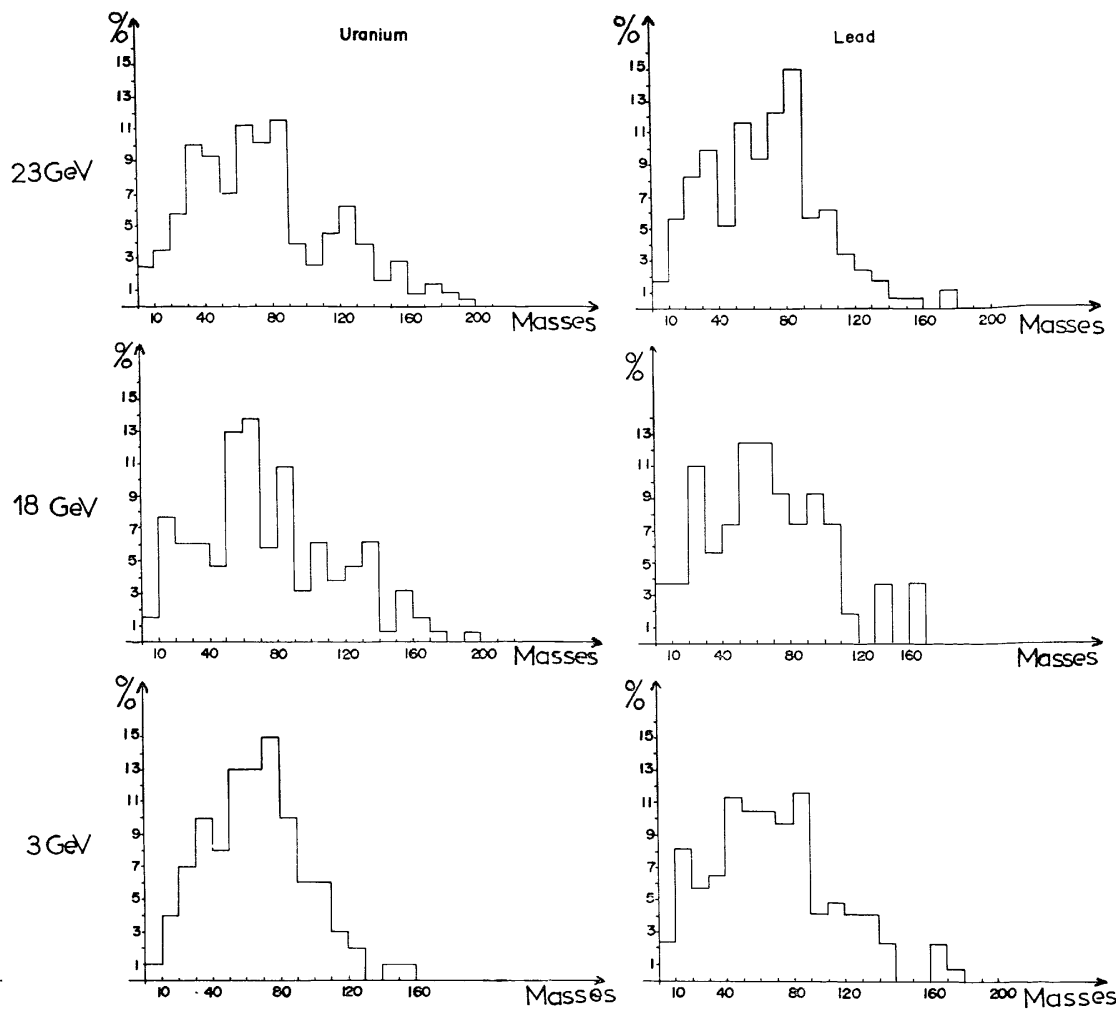

$\mathrm{b}$

FIG. 3.

a) High energy proton interactions with uranium, lead and gold targets ; ratio of ternary events to binary events.

b) Fragment mass distribution for coplanar events.

TABLE I

\begin{tabular}{|c|c|c|c|c|c|}
\hline \multirow{2}{*}{\multicolumn{2}{|c|}{ Cible }} & \multicolumn{4}{|c|}{ ÉNERGIE $(\mathrm{GeV})$} \\
\hline & & $\begin{array}{c}0.6 \\
-\end{array}$ & $\begin{array}{l}3 \\
-\end{array}$ & $\begin{array}{c}18 \\
-\end{array}$ & $\begin{array}{c}23 \\
-\end{array}$ \\
\hline \multirow{3}{*}{$\mathrm{U}$} & $T$ & 8 & 45 & 88 & 117 \\
\hline & $B$ & 18470 & 5702 & 4306 & 6787 \\
\hline & $T / B$ & $0.05(*)$ & $0.8 \pm 0.15$ & $2 \pm 0.3$ & $1.7 \pm 0.2$ \\
\hline \multirow{3}{*}{$\mathrm{Pb}$} & $T$ & 3 & 99 & 197 & 197 \\
\hline & $B$ & 2043 & 5456 & 5430 & 6572 \\
\hline & $T / B$ & $0.15(*)$ & $1.8 \pm 0.2$ & $3.6 \pm 0.4$ & $3 \pm 0.3$ \\
\hline \multirow{3}{*}{$\mathrm{Au}$} & $T$ & 16 & & 300 & 212 \\
\hline & $B$ & 1936 & & 4098 & 4249 \\
\hline & $T / B$ & $0.8\left(^{*}\right)$ & & $7.3 \pm 0.6$ & $5 \pm 0.5$ \\
\hline
\end{tabular}

(*) Indicates poor statistics. The represented error is only the statistical one.

Our numerical results are higher than those obtained by Hudis and Katcoff [8] and Brandt et al. [2]. This is due to the following reason :
- Makrofol is sensitive to particles of masses heavier than 16 (oxygen), but mica is sensitive only to masses with $M>30$. Thence our ternary fission events contain an important contribution of lighter fragments than those which can be visualized in mica.

The fact that $T / B$ increases with proton energy, and that, as it is well known, binary cross-section decreases slowly in the same conditions, confirmes the idea that ternary fission cross-section increases with incoming proton energy and this may be the result of a larger fragmentation contribution to this ternary process.

Mass calculation for ternary events. - We have taken a part of all found ternary fission events and made a first approach in calculating the mass and energy distribution for the particles emitted in ternary fission.

For this calculation one needs all geometrical parameters concerning these events, as well as an accurately determined range-energy relationship, well established 

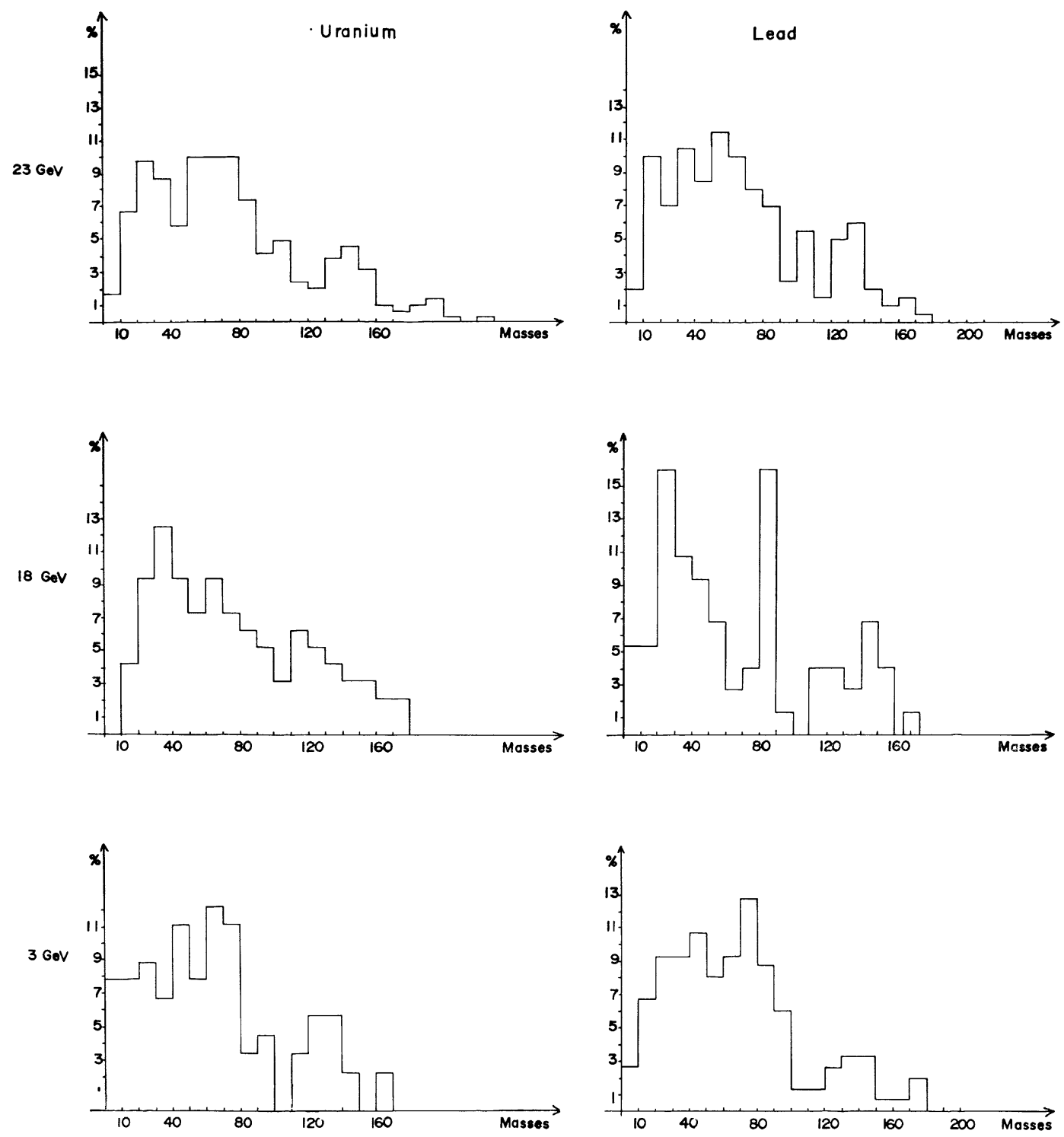

FIG. 4. - Fragment mass distribution for non coplanar events.

for heavy and lighter elements from common fission fragments to oxygen.

The projected ranges and the dip angles of the three prongs are measured with the help of an optical microscope. The error is $0.5 \mu$ for path measurements and $1 \mu$ for dip measurements. The projected azimuthal angles must also be determined $\left(\Delta \theta=2^{\circ}\right)$ for each track. The knowledge of these quantities, for every event, allows a spatial representation of each interaction. The largest experimental error is due to the dip measurements.

An other aspect is the necessity of getting a well determined range-energy relation for ions in our detector, as a function of their mass and charge numbers. This range-energy relation gives accurate results for masses between 32 and 160 A.M.U. However for low masses the formula has been found by extrapolation and is therefore inaccurately known. The closer to oxygen we are the larger is the mass-error.
This results from the fact that for lower masses, the theoretical relationship cannot be used because we are near the registration threshold in plastics.

We must point out, that for light ions registered in plastics, the imperfect knowledge of the range-energy relationship disturbs also the mass spectrum for heavier masses due to calculation procedures used here.

In this first rough calculation, we have assumed that $M / Z$ is equal to the $\beta^{-}$stability value given by the nuclear chart tables, instead of the classical well known E.C.D. or U.C.D. values. This approximation increases also the error in the resulting masses.

In our calculations, a hypothesis is needed concerning the mass of the fissioning nucleus. We have assumed in our calculations that the cascade and the evaporation take place before fission and lower the target mass for 10 A.M.U. (this arbitrarely choiced number is assumed to be independent of the incoming proton energy between 3 and $25 \mathrm{GeV}$ ). This assump- 

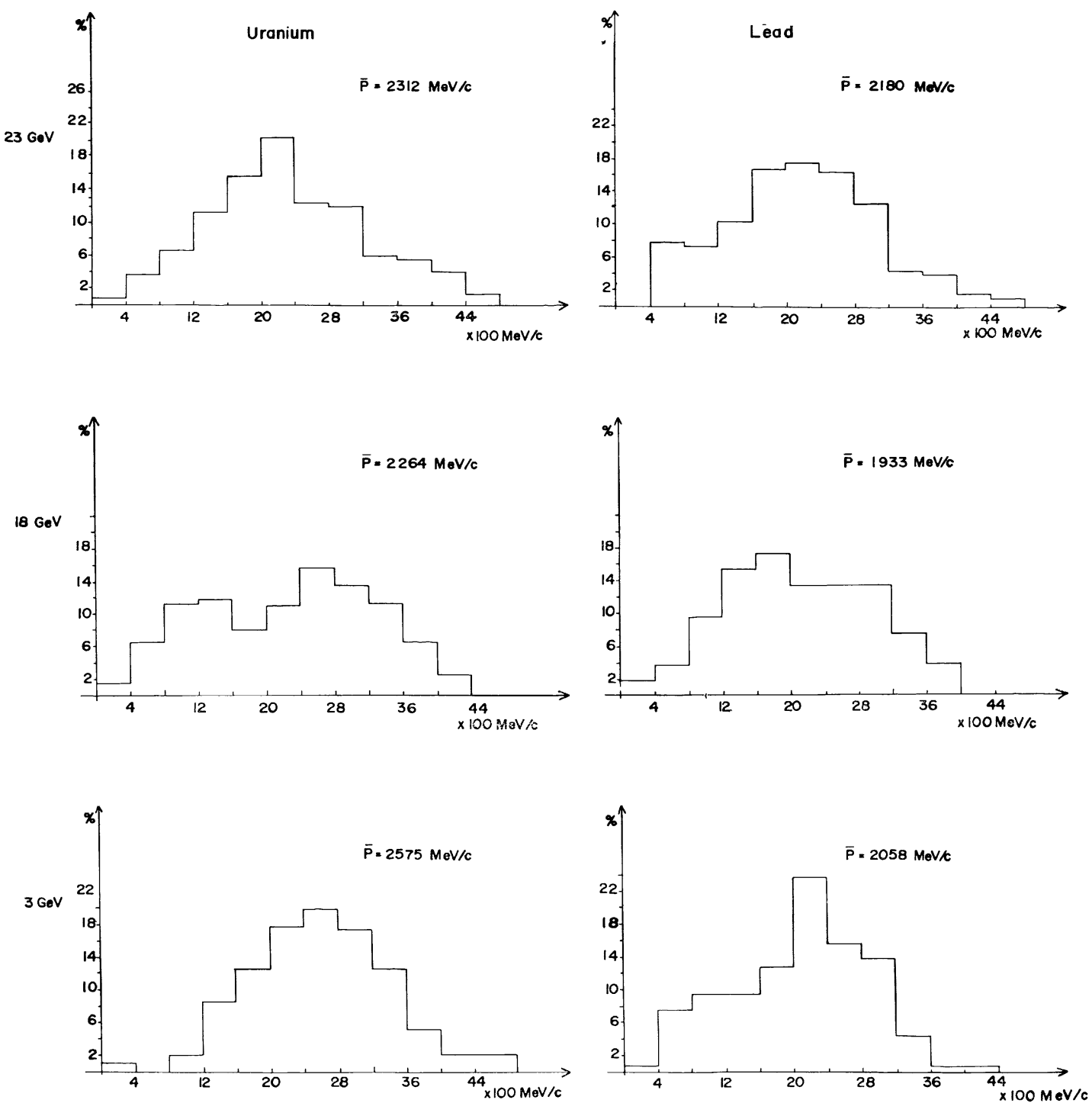

FIG. 5. - Fragment momentum distribution for coplanar events.

tion increases also the error we make in calculating masses.

But we must note that our method is the only possible one, which nowadays permits a mass determination for ternary events.

Presently, we are carring out new experiments concerning experimental range-energy determinations for visualized fragments in makrofol.

In future, when we have an accurate determination for the relation between range, energy, mass, and charge, we shall try an other calculation, where the assumption that the products are beta-stable will be eliminated. We may also take in our future calculation the fissioning mass as a parameter which will vary between certain limits, and see in this way the influence of this parameter on the mass results.

At 3 and at $23 \mathrm{GeV}$, we have observed a large number of events, but we must note that at $18 \mathrm{GeV}$, for technical reasons, our statistics are poor. Therefore, we regard the $18 \mathrm{GeV}$ results as less important.
We employed momentum and mass conservation laws for the mass calculation. The geometrical parameters of each event are determined experimentally. The following relations hold :

$$
\left\{\begin{aligned}
a_{1} P_{1}+a_{2} P_{2}+a_{3} P_{3} & =P_{x} \\
b_{1} P_{1}+b_{2} P_{2}+b_{3} P_{3} & =P_{y} \\
c_{1} P_{1}+c_{2} P_{2}+c_{3} P_{3} & =P_{z} \\
M_{1}+M_{2}+M_{3} & =A
\end{aligned}\right.
$$

$P_{x}, P_{y}, P_{z} \quad$ : the recoil momentum components of the moving nucleus,

$P_{1}, P_{2}, P_{3} \quad$ : the momentum of the three emitted particles,

$M_{1}, M_{2}, M_{3}$ : the corresponding masses,

$A \quad$ : the mass of the fissioning nucleus.

In order to solve these equations and to calculate $M_{1}$, $M_{2}, M_{3}$ and $P_{1}, P_{2}, P_{3}$, we need a well determined analytical mass momentum relationship. For the cal- 

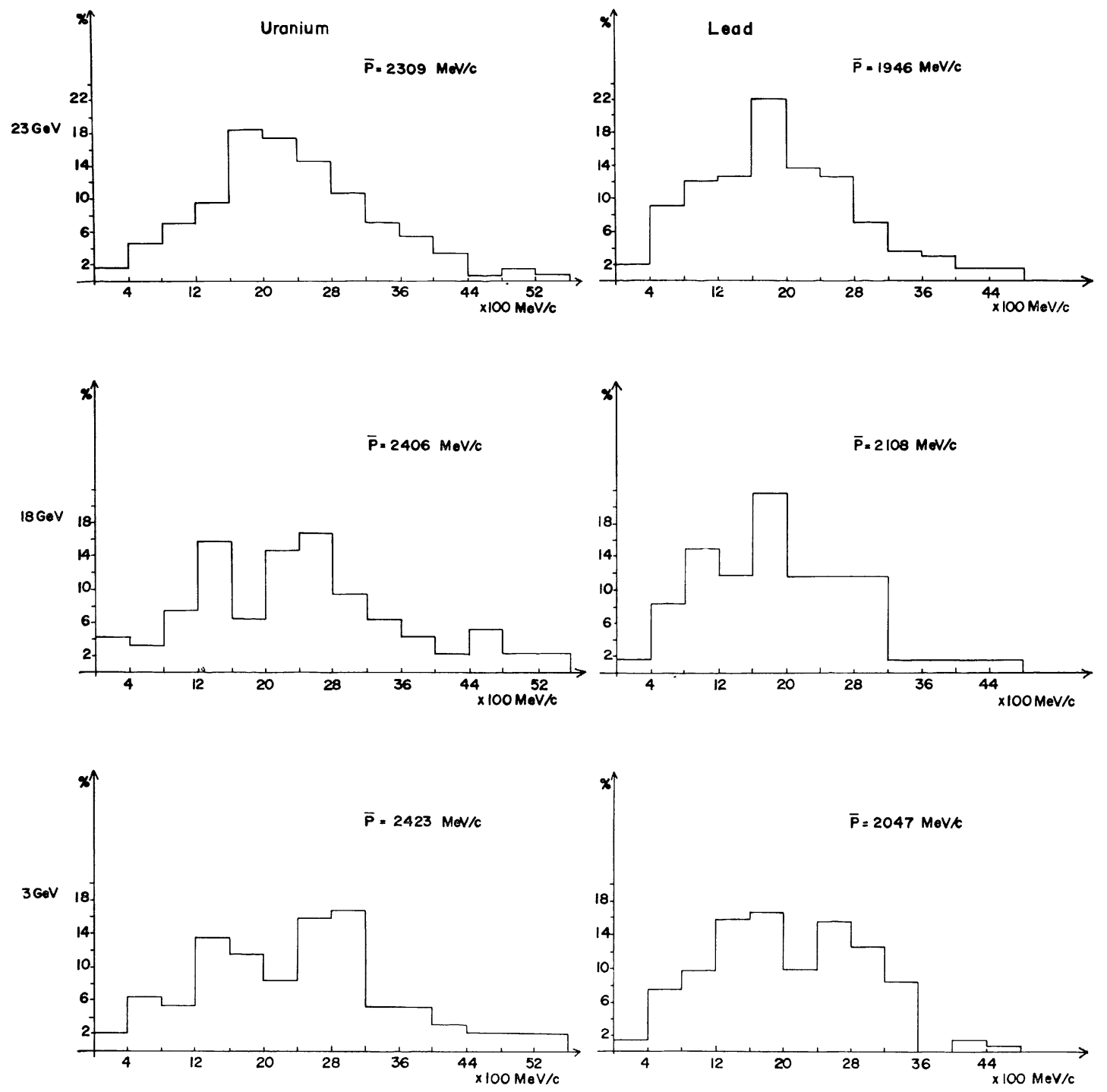

FIG. 6. - Fragment momentum distribution for non coplanar events.

culations, we have fitted the semi-experimental momentum-range-mass relationship by fourth deegre polynoms :

$$
\begin{aligned}
P_{\mathrm{i}}=\left(\alpha M_{\mathrm{i}}^{4}\right. & \left.+\beta M_{\mathrm{i}}^{3}+\gamma M_{\mathrm{i}}^{2}+\delta M_{\mathrm{i}}+\varepsilon\right) R_{\mathrm{i}} \\
& +\left(\alpha^{\prime} M_{\mathrm{i}}^{4}+\beta^{\prime} M_{\mathrm{i}}^{3}+\gamma^{\prime} M_{\mathrm{i}}^{2}+\delta^{\prime} M_{\mathrm{i}}+\varepsilon^{\prime}\right)
\end{aligned}
$$

$\left(P_{\mathrm{i}}=\right.$ momentum, $M_{\mathrm{i}}=$ the mass, $\left(\alpha, \beta, \gamma ; \alpha^{\prime}, \beta^{\prime}, \gamma^{\prime}\right)$ $=$ constants)), $R_{\mathrm{i}}$ is the range of the track in our detector, the computer is then able to resolve our equations (1). The following hypotheses have also been made :

1) In all calculations, we suppose that $P_{x}=P_{y}=0$, i.e. the recoil momentum is in the incoming direction.

2) We consider especially coplanar events, so that the recoil momentum is very small or zero, but the calculation has been performed with the same assumptions for all coplanar and non coplanar events.

It is very difficult to determine the errors in the mass calculations. Here are a few sources of error :

1) Experimental errors on path length measure- ments are of the order of $1 \mu$. This corresponds to 3 or 4 A.M.U. after calculation.

2) The error introduced by assuming $Z / M$ to be equal to the $\beta^{-}$stability value in range-energy determinations may be of the order of 1 A.M.U. for heavy fragments and 3 for lighter ones.

3) The uncertainty in our knowledge of the mass of the fissioning nucleus can lead to an error of 3 or 4 A.M.U. on each fragment mass.

To conclude, the total error in mass determinations is then roughly of the order of 10 A.M.U. For non coplanar events, the results are only given to be compared to coplanar ones. The results are shown in figures 3 to 8 .

We can note that :

1) With increasing proton energy the mass distributions becomes broader.

2) With increasing proton energy the velocity of the moving nucleus decreases.

3) The $F / B$ ratio decreases with increasing energy $\left(F / B\right.$ defined by the sign of $P_{z}$ while $\left.P_{x}=0 ; P_{y}=0\right)$. 

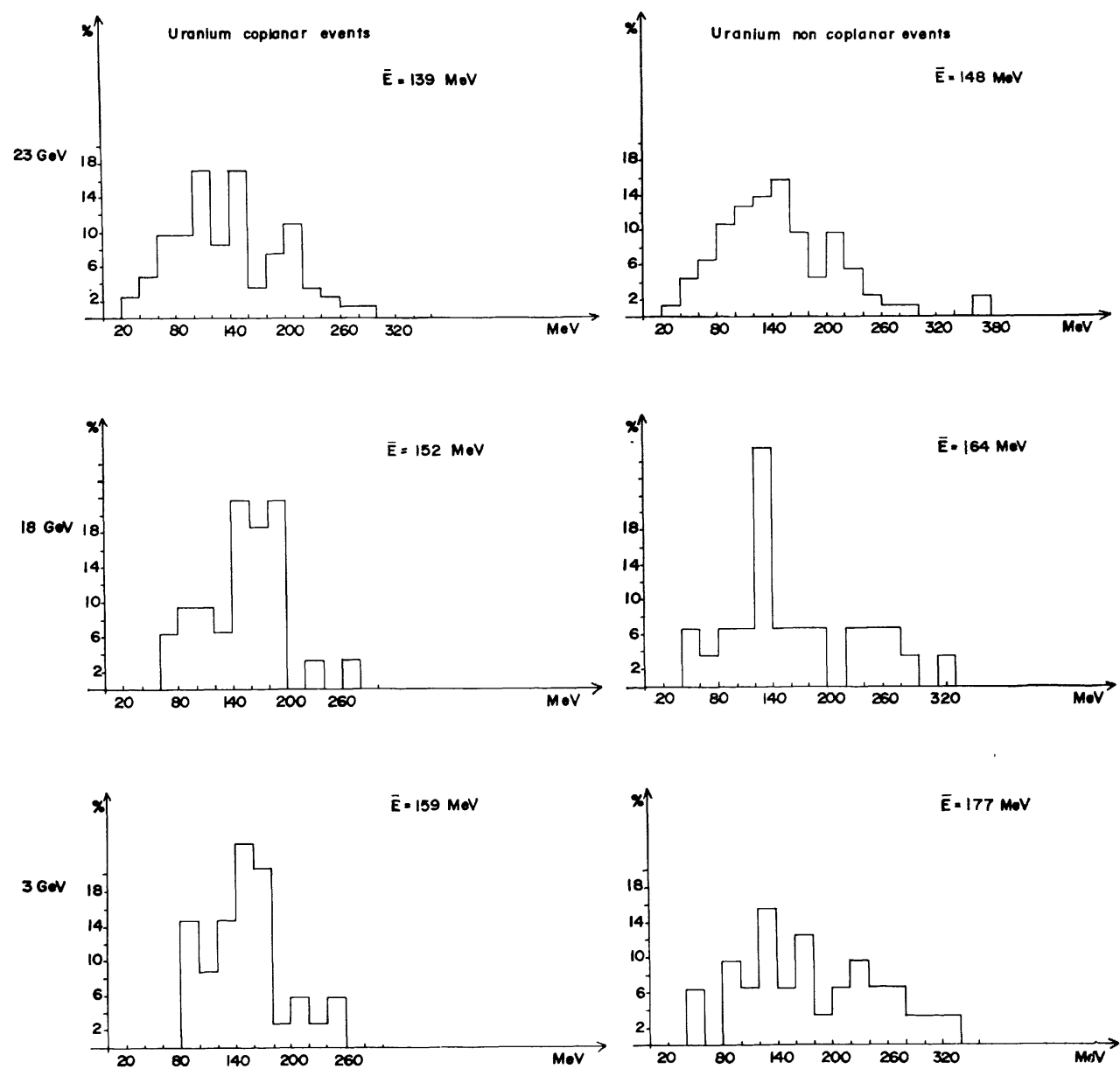

FIG. 7. - Total kinetic energy.
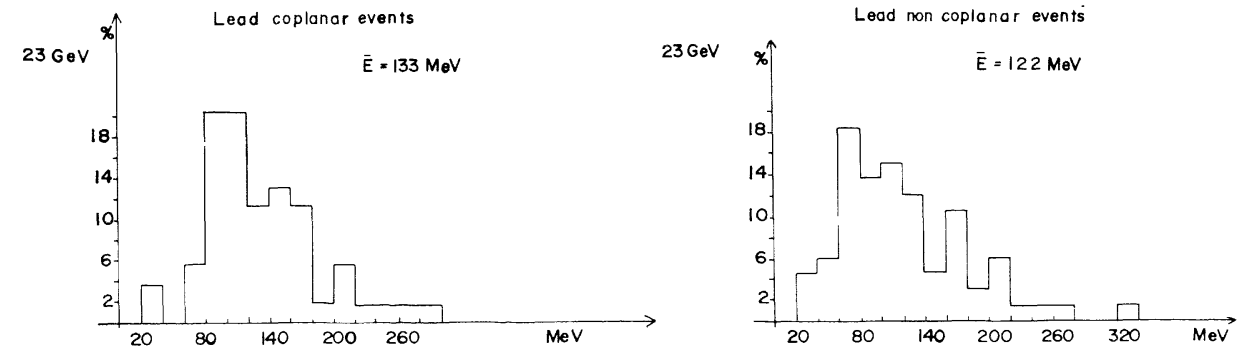

\begin{tabular}{c|c|c|c|c|}
\multicolumn{5}{c}{ Total mean kinetic energy in MeV } \\
$\begin{array}{c}\text { proton } \\
\text { GeV }\end{array}$ & $U$ copl & Unon copl & Pb copl & Pb non copl. \\
\cline { 2 - 5 } 23 & 139 & 148 & 133 & 122 \\
\cline { 2 - 5 } 18 & 152 & 164 & 120 & 107 \\
\hline 3 & 159 & 177 & 119 & 143 \\
\hline
\end{tabular}

FIG. 8. - Total kinetic energy. 

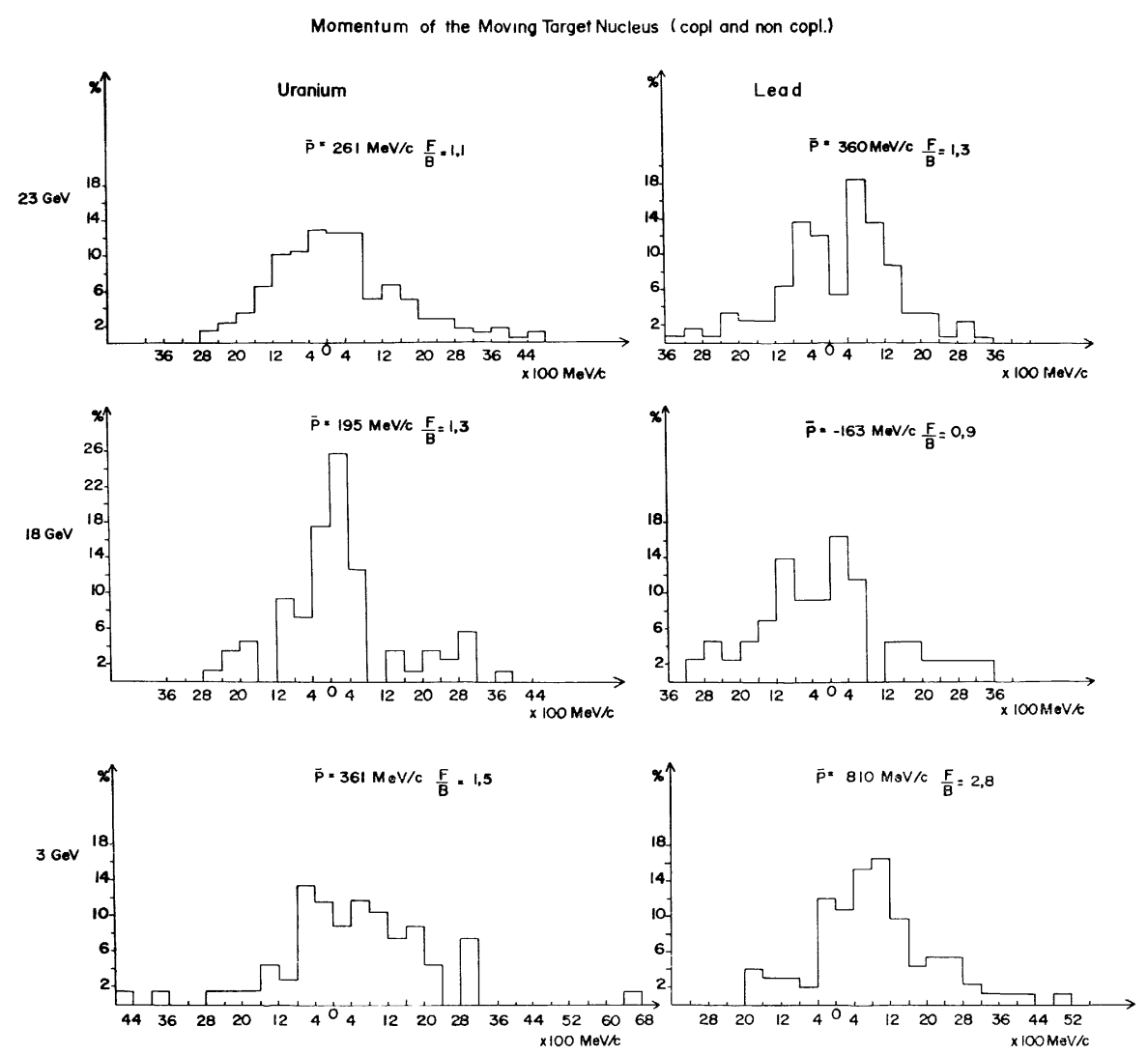

FIG. 9. - Momentum of the moving target nucleus (coplanar and non coplanar events).

Conclusion. - This paper was to provide only a general view on the use of plastic track detectors for the study of high energy fission. It is easy to determine accurately some relative cross-sections and mass and energy distributions. Rare events can be not only registered and counted but also studied kinematically, if one knows accurately the different geometrical parameters.

Concerning fission induced by charged particles, we plan to continue our work, especially in determinations of cross-sections of rare events and in calculations of mass and energy distribution.

Acknowledgements. - It is a pleasure to acknowledge many helpful discussions with our colleagues from C.E.R.N.-Heidelberg-Napoli-Warsaw collaboration (see preceding publication).

\section{BIBLIOGRAPHY}

[1] Debeauvais (M.), Stein (R.), Ralarosy (J.) and CÜER (P.), Nucl. Phys., 1967, A 90, 186-198.

[2] Brandt (R.), Carbonara (F.), Cieślak (E.), DakowSki (M.), GFelifer (Ch.), Piekarz (H.), Piekarz (J.), RIEZLER (N.), RINZIVILIO (R.), SASSI (E.), SoWINSKI (M.) and SAKRZEWSkI (J.), Nucl. Phys., 1967, A 90, 177-185.

[3] Dutra (S. P.), Ind. J. Phys., 1953, 27, 547.

[4] Ho (Z. W.), TSIEN (S. T.), Vigneron (L.) and ChasTEL (R.), C. R. Acad. Sci., 1946, 223, 1119 ; Phys. Rev., 1947, 71, 382.
[5] Muga (M. L.), Bowmann (H. R.) and ThompSoN (S. G.), Phys. Rev., 1961, 270.

[6] Fleischer (R. L.), Price (P. B.), Walker (R. M.) and Hubbard (E. L.), Phys. Rev., 1966, 143, 943.

[7] Hyde (E.), Nuclear Properties of the Heavy Elements, vol. III, p. 131 (Prentice Hall, Inc., Englewood Cliffs/New Jersey).

[8] Hudis (J.) and KATCOFF (S.), BNL 13124 (submitted to Phys. Rev., 1969). 